\title{
Increased Permeability of the Aquaporin SoPIP2;1 by Mercury and Mutations in Loop A
}

\author{
Andreas Kirscht', Sabeen Survery ${ }^{\dagger}$, Per Kjellbom and Urban Johanson* \\ Department of Biochemistry and Structural Biology, Center for Molecular Protein Science, Lund University, Lund, Sweden
}

Aquaporins (AQPS) also referred to as Major intrinsic proteins, regulate permeability of biological membranes for water and other uncharged small polar molecules. Plants encode more AQPs than other organisms and just one of the four AQP subfamilies in Arabidopsis thaliana, the water specific plasma membrane intrinsic proteins (PIPs), has 13 isoforms, the same number as the total AQPs encoded by the entire human

OPEN ACCESS

Edited by:

Rupesh Kailasrao Deshmukh, Laval University, Canada

Reviewed by: Gunvant Baliram Patil, University of Missouri, USA Dirk Schneider, University of Mainz, Germany Micaela Carvajal, Spanish National Research Council, Spain

*Correspondence:

Urban Johanson urban.johanson@biochemistry.lu.se

tThese authors shared first authorship, names in alphabetical order.

Specialty section: This article was submitted to Plant Physiology,

a section of the journal Frontiers in Plant Science

Received: 29 May 2016 Accepted: 08 August 2016 Published: 30 August 2016

Citation: Kirscht A, Survery S, Kjellbom P and Johanson U (2016) Increased Permeability of the Aquaporin SoPIP2; 1 by Mercury and Mutations in Loop A. Front. Plant Sci. 7:1249. doi: 10.3389/fpls.2016.01249 genome. The PIPs are more conserved than other plant AQPs and here we demonstrate that a cysteine residue, in loop A of SoPIP2; 1 from Spinacia oleracea, is forming disulfide bridges. This is in agreement with studies on maize PIPs, but in contrast we also show an increased permeability of mutants with a substitution at this position. In accordance with earlier findings, we confirm that mercury increases water permeability of both wild type and mutant proteins. We report on the slow kinetics and reversibility of the activation, and on quenching of intrinsic tryptophan fluorescence as a potential reporter of conformational changes associated with activation. Hence, previous studies in plants based on the assumption of mercury as a general AQP blocker have to be reevaluated, whereas mercury and fluorescence studies of isolated PIPs provide new means to follow structural changes dynamically.

Keywords: aquaporin, water channel, major intrinsic protein, Spinacia oleracea, tryptophan fluorescence

\section{INTRODUCTION}

Aquaporins (AQPs) are common in all domains of life and facilitate permeation of a wide range of small polar molecules through biological membranes (Abascal et al., 2014). AQPs are generally found as homotetramers, where each monomer constitutes a separate pore formed by six transmembrane helices and two short helices that are connected in the middle of the lipid bilayer at their N-termini (Fu et al., 2000; Sui et al., 2001; Törnroth-Horsefield et al., 2006; Horsefield et al., 2008; Kirscht et al., 2016). Members belonging to the plant subfamily of plasma membrane intrinsic proteins (PIPs) are specifically permeable to water and show a more strict amino acid sequence conservation than AQPs in other subfamilies (Danielson and Johanson, 2010). Function and abundance of these proteins is tightly regulated and the high number of isoforms suggests a highly redundant system for water homeostasis (Johanson et al., 2001; Alexandersson et al., 2005, 2010). Structure and regulation of one particular member of the PIP subfamily from spinach (Spinacia oleracea), SoPIP2;1, which constitutes a dominating integral protein of the plasma membrane has been thoroughly studied (Johansson et al., 1996, 1998; Kukulski et al., 2005; Törnroth-Horsefield et al., 2006; Nyblom et al., 2009; Frick et al., 2013). 
The structure of SoPIP2;1 was solved in different conformations, which elucidated a general molecular gating mechanism for the regulation of PIPs, including some specific elements only relevant for members of the PIP2 subgroup (Törnroth-Horsefield et al., 2006). The gating of the pore is the result of conformational changes on the cytoplasmic side of the membrane and is controlled in several different ways, including, $\mathrm{pH}$ changes, binding of calcium and a PIP2 specific phosphorylation. The second cytosolic loop, Loop D, undergoes a major conformational change and its stabilization in certain positions is increasing the probability of an either open or closed conformation. In the closed conformation, unphosphorylated serine 274, situated close to the C-terminus, occupies a place near the tetrameric center of the protein while the preceding region resides in a grove between the monomers. When this positioning is impossible, e.g., by phosphorylation of serine 274, the C-terminus becomes unordered and the place earlier occupied by this residue is instead taken by the carbonyl oxygen of leucine 197 from the neighboring monomer, resulting in the unblocking of the pore in this monomer.

Already in early protein preparations, it became clear that interactions between monomers are strong in SoPIP2;1 tetramers (Johansson et al., 1996). Even during separation by a standard SDS-PAGE, which denatures most proteins, a large fraction of the protein is found to stay as dimers and tetramers (Karlsson et al., 2003). PIPs differ from all other plant AQPs by a highly conserved cysteine in the first extracellular loop (loop A). The conserved cysteines (C69 in SoPIP2;1) from all four monomers are located at the tetrameric center (Törnroth-Horsefield et al., 2006). Based on low resolution structures, it had been suggested that the reason for the conservation stems from the necessity to stabilize the PIP tetramers by fostering hydrogen bonds or complexing a metal ion (Kukulski et al., 2005).

To analyze the nature of possible interactions of the conserved cysteine (C69) in the tetramer, we probed possible disulfide bridges by addition of reducing agents. For further analysis, we created mutants and purified the heterologously expressed proteins. In this study, we measured stability changes for cysteine 69 mutants and compared these to their kinetic properties to elucidate the structural and functional basis for the strong evolutionary conservation of this amino acid residue. In addition we show that mercury - generally regarded as a blocker of AQPs - is activating SoPIP2;1 and concentration-response experiments suggest that quenching of tryptophan fluorescence reports on conformational changes associated with the activation. Our findings of disulfide bridges and mercury activation are largely consistent with results recently published by other groups (Bienert et al., 2012; Frick et al., 2013).

\section{RESULTS}

\section{A Conserved Cysteine Forms Disulfide Bridges between Monomers}

To discern inter-monomeric interactions of SoPIP2;1 further, we decided to study structural and functional properties of isolated wild type (WT) and mutant protein. Therefore, we overexpressed SoPIP2;1 in Pichia pastoris and performed different stability tests of solubilized and purified protein. The isolated WT protein was incubated for various length of time with different reducing agents prior to analysis by SDS-PAGE, in order to monitor any effect of potentially disrupted disulfide bridges on the migration pattern in acrylamide gels (Figure 1). The relative amount of SDS resistant dimer in the gel could be decreased by DTT and $\beta$-mercaptoethanol. The latter was more effective, but none of the reducing agents could increase the monomer-to-dimer ratio further after the first hour of incubation. Surprisingly, the split of the dimer was not increased above $100 \mathrm{mM}$ DTT and the final ratio of monomer to dimer was less than $1 / 10$, while $\beta$-mercaptoethanol decreased the dimers by $50 \%$ at most (not
A

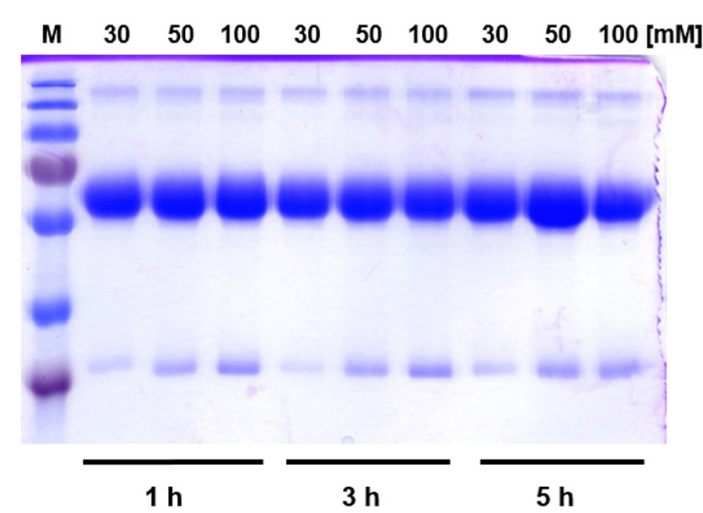

B

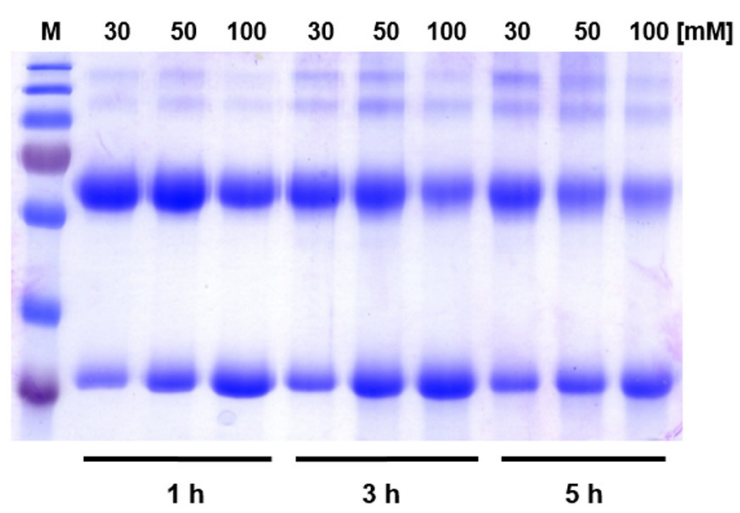

FIGURE 1 | The effect of reducing agents on relative amounts of dimers and monomers of SoPIP2;1. (A) Incubation of SoPIP2;1 with DTT. The concentration of DTT and the incubation time are shown at the top and at the bottom of the gel, respectively. It is evident from the gel that DTT is unable to completely reduce dimers into monomers, even after $5 \mathrm{~h}$ incubation with $100 \mathrm{mM}$ DTT. (B) Effect of incubation of SoPIP2; 1 with $\beta$-mercaptoethanol. The concentration of $\beta$-mercaptoethanol and the incubation time are shown on top and bottom of the gel, respectively. The results show that $\beta$-mercaptoethanol is more effective than DTT, but it still fails to reduce the dimers into monomers completely. $\mathrm{M}=$ molecular weight marker (from top $250,130,100,70,55,35$, and $25 \mathrm{kDa}$ ). 


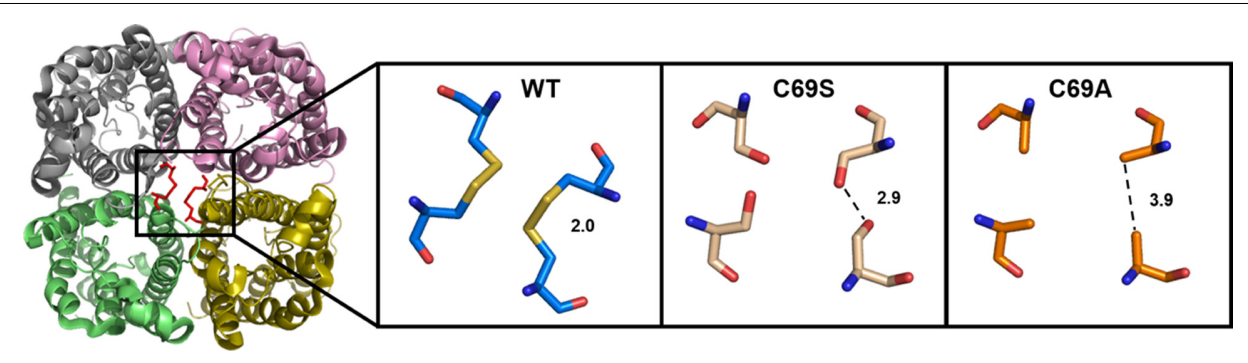

FIGURE 2 | Extracellular view of the SoPIP2;1 wild type (WT) tetramer with the expected disulfide bridges at the center (Left). Close up on C69 with distances between cysteines (PDB ID: 2B5F) or modeled residues in mutant proteins with this cysteine replaced by serine (Middle) or alanine (Right).

shown). Either this reflects the equilibrium between monomeric and dimeric fraction when all disulfide bonds are reduced, mainly stabilized by hydrophobic interactions (Törnroth-Horsefield et al., 2006), or some of the SDS solubilized dimers have disulfide bonds that are inaccessible so that they cannot be reduced under these conditions. The latter is supported by the apparent higher efficiency of $\beta$-mercaptoethanol which has about half the molecular weight compared to DTT. Adding urea to the reduction step increased the monomeric fraction (not shown). However, not even incubation for $1 \mathrm{~h}$ with $8 \mathrm{M}$ urea and $300 \mathrm{mM}$ DTT and resolving the samples on an $8 \mathrm{M}$ denaturing urea gel was sufficient to completely remove the multimeric bands (Supplementary Figure 1). From a physical and evolutionary point of view it is of interest if the stability of the protein is changed upon removal of the cysteine. Two mutants, substituted at cysteine 69 with serine (C69S) or alanine (C69A) (Figure 2), were expressed and purified and their oligomeric patterns (as shown by standard SDS-PAGE) were compared to the WT protein (Figure 3A). Although dimeric bands were still visible in both mutants, they were significantly weaker as compared to the WT protein, which was expected from the destabilizing effect of reducing agents on dimers of the WT protein. In order to compare the thermodynamic stability of WT SoPIP2;1 and the two cysteine 69 mutants, we performed thermal denaturation circular dichroism (CD) spectroscopic measurements. A thermal denaturation curve was constructed based on the normalized mean residual ellipticity (MRE) at $222 \mathrm{~nm}$ (Figure 3B). The midpoint of transition in WT $\left(56.9^{\circ} \mathrm{C}\right)$ was slightly higher in comparison with the mutants $\left(54.6^{\circ} \mathrm{C}\right.$ for $\mathrm{C} 69 \mathrm{~S}$ and $53.4^{\circ} \mathrm{C}$ for $\mathrm{C} 69 \mathrm{~A}$ ), suggesting that the interactions at this position do not contribute much toward the thermodynamic stability, which is expected since the contribution of disulfide bonds to protein stability is kinetic rather than thermodynamic (Clarke and Fersht, 1993). Considering the high stability of SoPIP2;1 in lipid bilayers (Plasencia et al., 2011), it is not clear if the relatively small additional thermal stabilization due to the disulfide bonds is sufficient to explain the strict conservation of this cysteine.

\section{Water Permeability Is Increased by Mutations in Loop A}

To investigate if the disulfide bonds have an effect on function all three proteins were reconstituted in proteoliposomes to examine

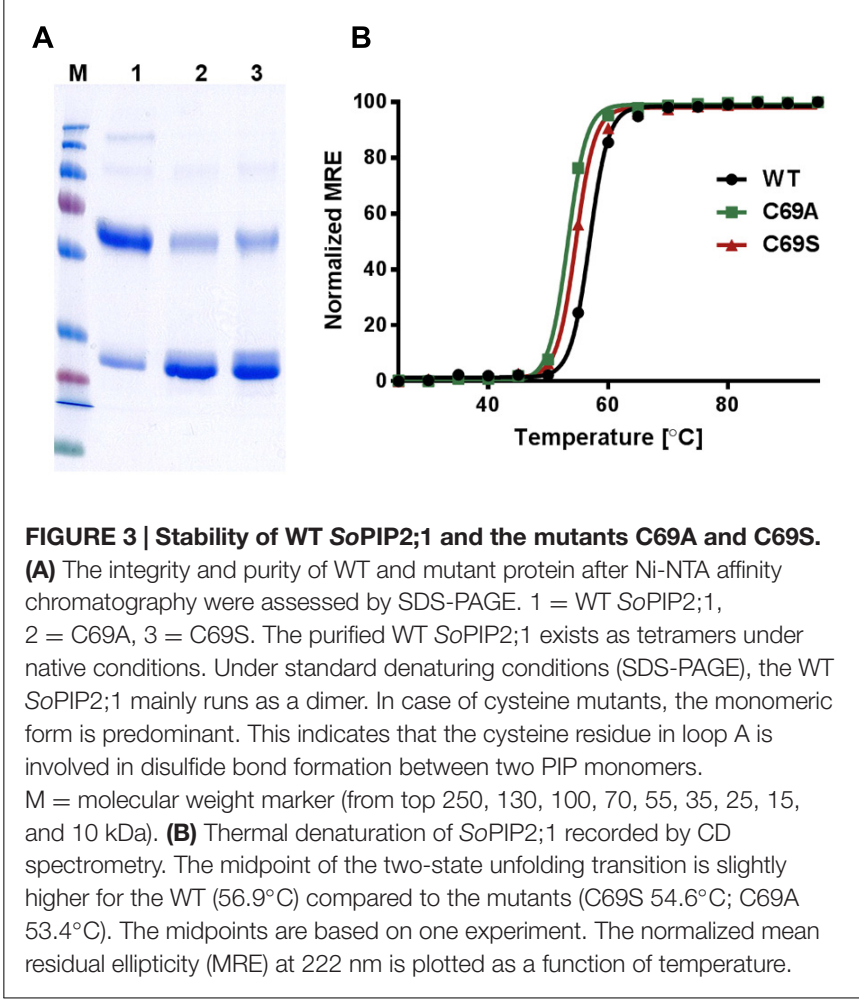

their kinetic properties. Since the activity assays confirmed functional proteins (Figure 4A), we assessed their permeabilities. The protein content in the liposomes was estimated by quantitative western blots and the monomeric permeabilities, the $p_{\mathrm{f}}$-values, were calculated. Surprisingly, the mutant C69S shows increased water permeability $(P<0.001)$ and this is even more pronounced in the C69A mutant $(P<0.0001$; Figure 4B). The location of this residue immediately raises the question about water transport through the central pore. This scenario would be consistent with the observed larger enhancement of water permeability when cysteine is exchanged with an amino acid residue smaller than serine. Alternatively the increase is purely caused by destabilization of the dimer, where the alanine mutant would not be able to form hydrogen bonds like the serine mutant, which may partially compensate for the missing disulfide bridges. 
A



B

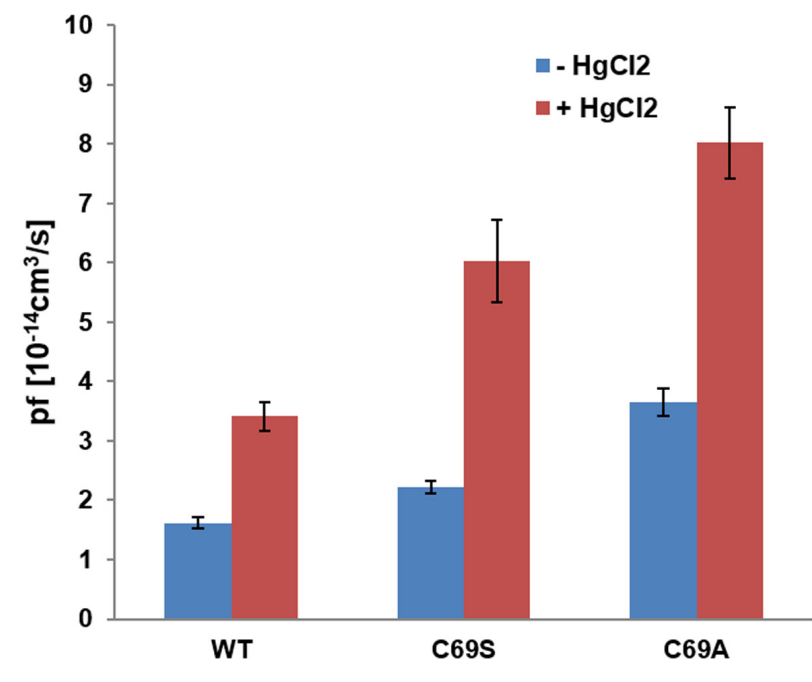

FIGURE 4 | Water conductivity of liposomes measured by stopped-flow spectrometry. (A) Change in scattering at 500 nm over time plotted for SoPIP2;1 WT and C69 mutant proteoliposomes as compared to empty liposomes. Each plot represents an average of 10-17 curves. (B) Monomeric permeabilities without mercury (blue) and with $1 \mathrm{mM}$ mercury (red). The rates were obtained from single exponential fits of 10 or more curves; error bars present their standard deviations. The water conductivities were higher for the mutants as compared to the WT protein and mercury increased the $p_{f}$-values of all proteins by a similar factor (2.1-2.7). The rate constants and the $p_{f}$-values are provided in Supplementary Table 1, while the statistics for comparisons of the $p_{f}$-values are reported in Supplementary Table 2.

\section{Mercury Increases Water Permeability and Quench Intrinsic Tryptophan Fluorescence}

If some water permeation is independent of the pore in the monomer, it could be measured by eliminating the conductivity of the monomeric pore. Beside reported $\mathrm{pH}$ and $\mathrm{Ca}^{2+}$ dependent gating, mercury is a well-known inhibitor of AQPs (Preston et al., 1993; Hukin et al., 2002). To our surprise, mercury instead increased the permeability of SoPIP2;1 proteoliposomes $(P<0.0001$; Figure 4). If this effect is a result of conformational changes, there is a chance to observe mercury binding by CD measurements or by monitoring the intrinsic tryptophan fluorescence of SoPIP2;1. The CD spectrum of SoPIP2;1 in detergent micelles did not change significantly upon the addition of mercury (data not shown). However, the tryptophan fluorescence was altered and quenched by a factor of three after an incubation with $200 \mu \mathrm{M}$ mercury chloride (Figure 5). Moreover, the quenching could be reversed by chelating mercury with $\beta$-mercaptoethanol, supporting that it is caused by reversible conformational changes rather than denaturation of SoPIP2;1. This is in accordance with the kinetic experiments, where mercury activation can be reversed by addition of $\beta$-mercaptoethanol (Figure $\mathbf{6 A}$ ). It should be noted that the concentrations of reducing agent used for the reversal of quenching and activation, 1 and $2 \mathrm{mM}$, respectively, are much lower than the levels required to partially reduce the disulfide bridge of the dimers. Thus given the modest increase in activity of the C69S mutant completely lacking the disulfide bridge, any increase due to reduction of cysteine 69 is expected to be small. This is consistent with the more or less identical rates of untreated proteoliposomes and the activated and reversed ditto. To investigate the concentration dependency of the activation stopped-flow experiments were done and the $\mathrm{EC}_{50}$ for mercury was calculated to be around $4 \mu \mathrm{M}$ (Figure 6B). Interestingly, a concentration-response curve of the quenching of tryptophan fluorescence gives an $\mathrm{EC}_{50}$ value of $1.6 \mu \mathrm{M}$ (Figure 7). Thus the quenching in micelles may be caused by conformational changes associated with activation in proteoliposomes.

\section{Activation by Mercury and by Loop A Mutations Are Additive}

Cysteine 69 mutants present an increased water transport compared to the WT, which might be explained by opening of a tetrameric central pore or by indirect effects on the structure of the monomeric pore. WT SoPIP2;1 can be activated by mercury, but are the cysteine mutants also affected by mercury? Analysis of stopped-flow data, showed that the activation by mercury and the increase of permeability by mutations were not overlapping, but additive (Figure 4B). While the $p_{\mathrm{f}}$ of the WT permeability changes from $1.6 \pm 0.1$ to $3.4 \pm 0.2\left[10^{-14} \mathrm{~cm}^{3} / \mathrm{s}\right]$, the $p_{\mathrm{f}}$ of alanine and serine mutants are amplified by about the same factor, rising from $3.6 \pm 0.2$ to $8.0 \pm 0.6$ and $2.2 \pm 0.1$ to $6.0 \pm 0.7\left[10^{-14} \mathrm{~cm}^{3} / \mathrm{s}\right]$, respectively. This suggest that the mercury activation is independent of the increase in water permeability caused by the mutations in loop A. 
A

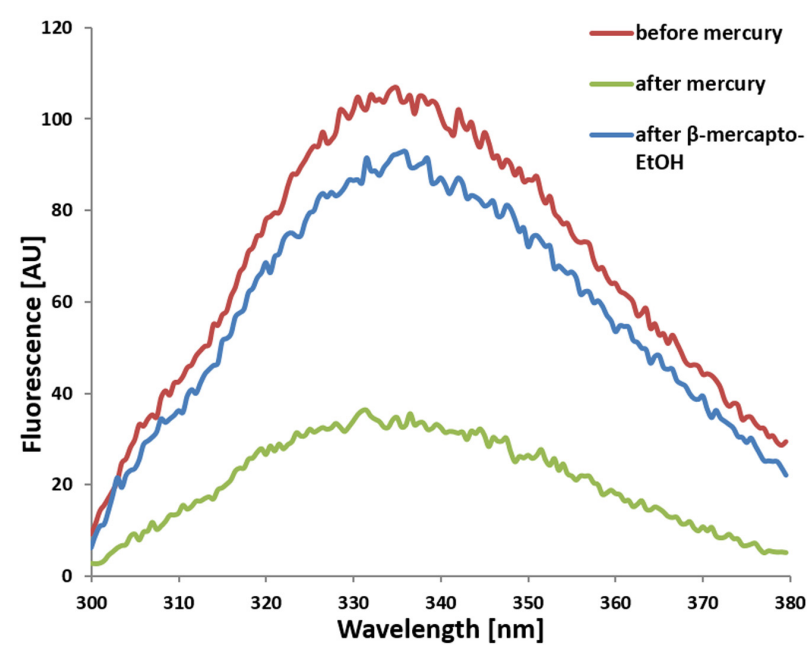

B

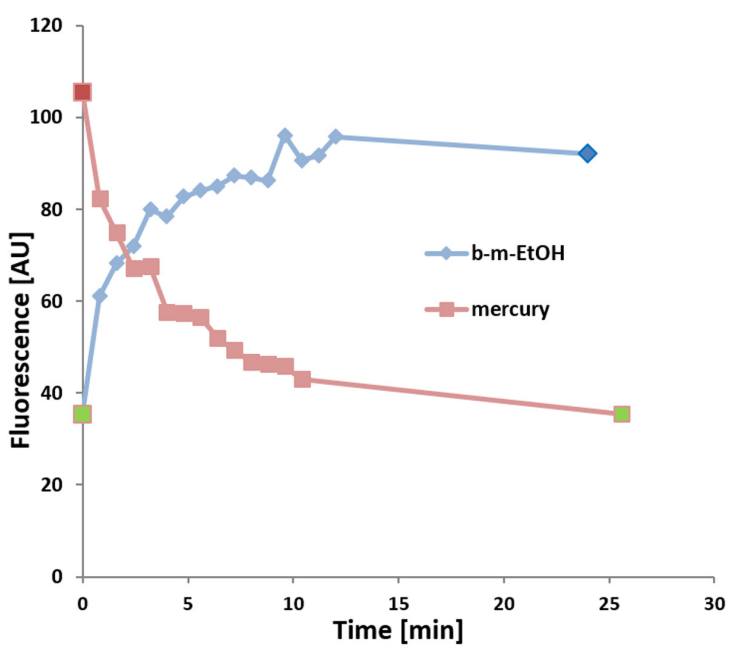

FIGURE 5 | (A) Quenching of tryptophan fluorescence of WT SoPIP2;1 upon addition of $200 \mu \mathrm{M}$ mercury (green) and subsequent reversal by addition of $1 \mathrm{mM}$ $\beta$-mercaptoethanol (blue) in comparison to untreated protein (red). The fluorescence at $335 \mathrm{~nm}$ from excitation at $280 \mathrm{~nm}$ is lowered by two thirds. (B) Time dependence of fluorescence quenching upon addition of $200 \mu \mathrm{M} \mathrm{HgCl}_{2}$ (light red) and recovering of fluorescence upon subsequent addition of $1 \mathrm{mM}$ $\beta$-mercaptoethanol to the same sample (b-m-EtOH, light blue). The individual maximum of every spectrum are plotted, where each spectrum was recorded at a scanning speed of $100 \mathrm{~nm} / \mathrm{min}$ (48 s per spectrum $+1 \mathrm{~s}$ delay). The spectra in (A) correspond to the three time points marked in the same color in (B): before addition of $\mathrm{HgCl}_{2}$ (red), after 25 min incubation with $\mathrm{HgCl}_{2}$ but before addition of $\beta$-mercaptoethanol (green; replotted at time zero), and at the endpoint after the incubation with $\beta$-mercaptoethanol (blue).
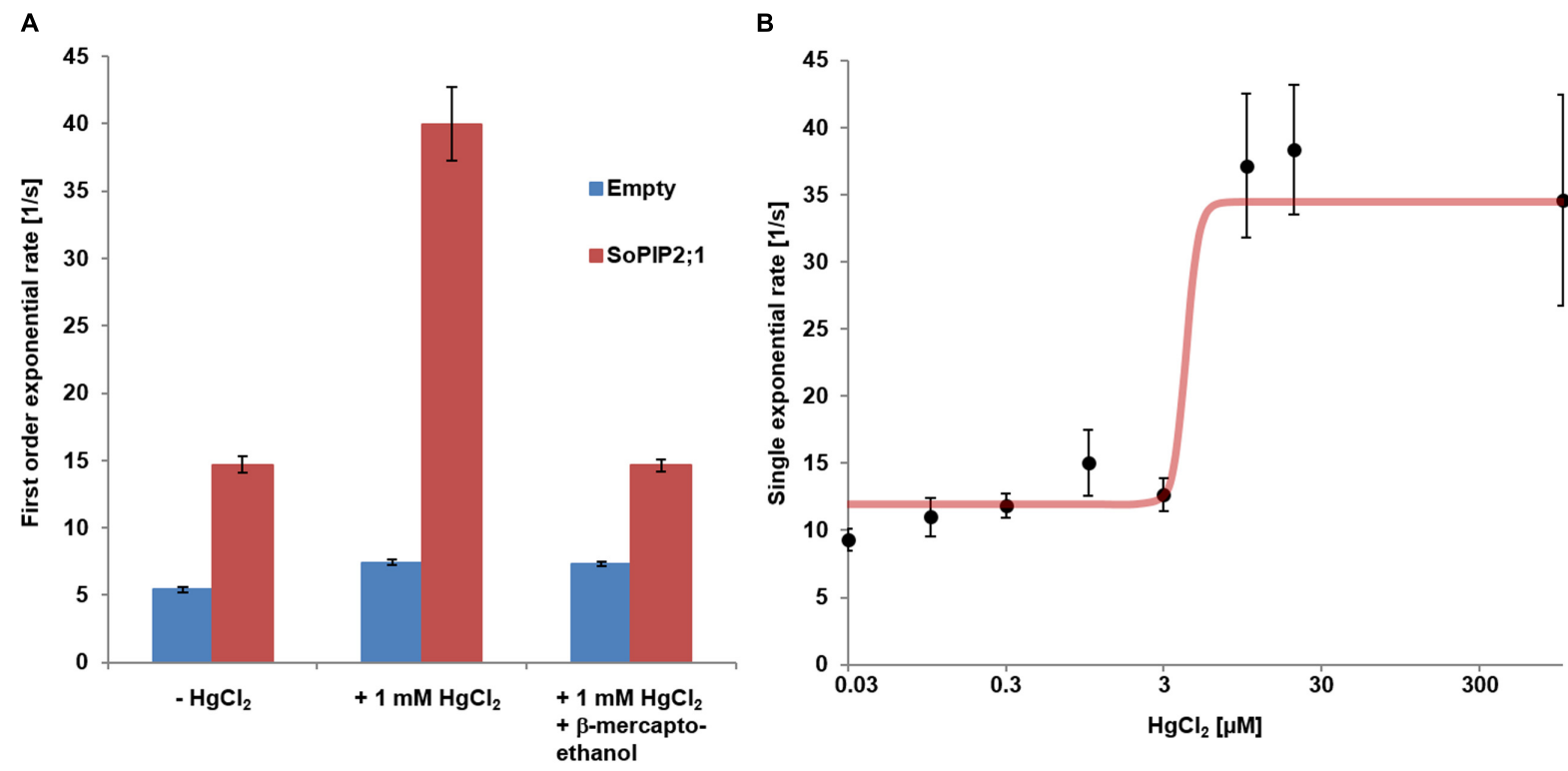

FIGURE 6 | Mercury activation of WT SoPIP2;1. (A) Reversibility of mercury activation first order exponential rates of water conductivity from stopped-flow experiments. Mean rates $( \pm S D)$ are presented for proteo-liposomes (red) and control liposome (blue). Activation of proteo-liposomes treated with mercury can be reversed by subsequent incubation with $2 \mathrm{mM} \beta$-mercaptoethanol. Statistics for comparisons of the rate constants are reported in Supplementary Table 3 and numerical values for mean rates and SD are found in Supplementary Table 4. (B) Dose response curve of mercury activation. The curve presents a Hill coefficient around 11 and an $\mathrm{EC}_{50}$ for mercury of $4.2 \mu \mathrm{M}$.

\section{DISCUSSION}

This work was originally initiated to describe intermonomeric interactions and their effects. The effect of reducing agents on the oligomeric pattern in SDS-PAGE indicated the existence of disulfide bonds that stabilize protein dimers. This was also concluded in studies mutating corresponding cysteines in the tetrameric center of various PIP2s from 


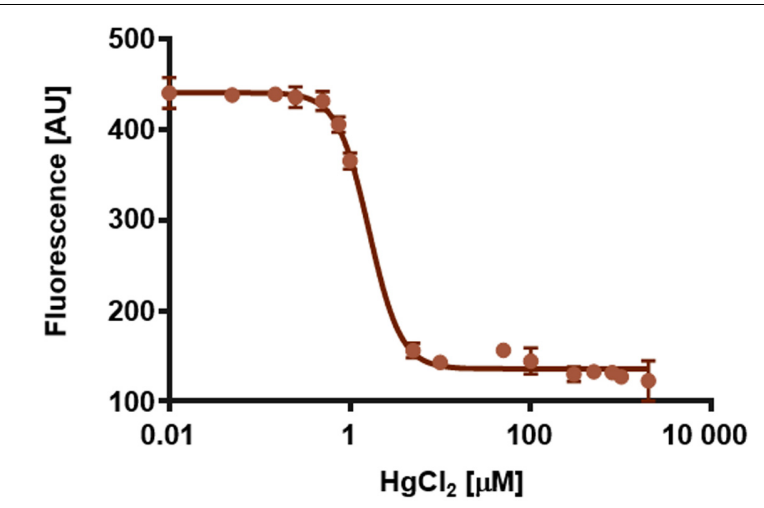

FIGURE 7 | Quenching of the intrinsic tryptophan fluorescence of SoPIP2;1 at $335 \mathrm{~nm}$ as function of the logarithm of the $\mathrm{HgCl}_{2}$ concentration. The calculated $\mathrm{EC}_{50}$ is $1.6 \mu \mathrm{M}$ and the Hill slope is -2.5 . Averages of three replicates and standard errors are plotted.

maize (Bienert et al., 2012). Additional to the more detailed analysis of disulfide bridges in the tetrameric center, we discovered that our preparation of SoPIP2;1 was not maximally permeable under our standard conditions. It should be noted that the obtained $p_{\mathrm{f}}$-values here (1.6-8.0 $\left.\left[10^{-14} \mathrm{~cm}^{3} \mathrm{~s}^{-1}\right]\right)$ are in the same range as has been estimated for other AQPs using Xenopus oocytes $\left(0.2-24\left[10^{-14} \mathrm{~cm}^{3} \mathrm{~s}^{-1}\right]\right.$; Yang and Verkman, 1997) and correspond to a passage of more than $10^{11}$ water molecules per second through each monomer of SoPIP2;1. Although activation of a water specific channel by mercury is unlikely to have a physiological role, the molecular details of its binding and conformational changes may still be relevant for our understanding of physiological gating mechanisms.

\section{Increased Permeability in Mutants and by Mercury}

Our results suggest that the mechanism for activation by mercury is independent of the enhancement of water permeability caused by the mutations in the A loop. A simple mechanistic explanation of this would be that permeation through the monomeric pore is increased in the mutants and this is amplified by mercury by stabilization of the gate in an open conformation. However, looking at the structure it is not obvious how a mutation of cysteine 69 could achieve this, but it cannot be excluded that the release of loop $\mathrm{A}$ is resulting in a relaxation at the selectivity filter and thereby a higher permeability. If instead also the central pore of the mutant proteins permeates water, how would that be enhanced by mercury binding? Possibly in the same way mercury may support the open conformation of loop $\mathrm{D}$ at the monomeric pore; the C-terminal end is removed from its position in the inter-monomeric space. If the highest energy barrier for water permeation through the central pore is caused by interactions between the four C-termini, a destabilization of the C-terminal regions would increase the water leakage through this hypothetical pore. One should keep in mind that these
C-terminal interactions are not structurally supported as the last modeled amino acid residues in the closed structures are still too far from the center. Thus a possible contribution of the central pore to the increased permeability remains speculative.

\section{Tryptophan Fluorescence}

The observation that both activation and quenching of tryptophan fluorescence can be triggered by the same ligand, mercury, may suggest that these two effects are not independent but caused by structural rearrangements initiated from the same binding site. So where is the mercury binding and which conformational changes lead to a fluorescence drop of $2 / 3$ ? If all six tryptophans of the monomer have the same quantum yield, at least four of them must be quenched. That involves a huge structural change of the protein or putatively a rearrangement of the micelle. Restructuring the micelle of a membrane protein is thought to denature the protein, which is unlikely here, as quenching (Figure 5) and increase in activity (Figure 6A) are both reversible, and no denaturation was indicated in $\mathrm{CD}$ measurements or by precipitating protein (data not shown). Fluorescence intensity of tryptophans can vary greatly depending on their environment (Lakowicz, 2006). Therefore, it appears more likely that one or two tryptophans with high quantum yields, located in a hydrophobic/hydrophilic interface, are responsible for the observed quenching of fluorescence by mercury. According to analyses of structural parameters tryptophans can be classified into five spectral-structural classes (Shen et al., 2008). The observed emission maximum at $335 \mathrm{~nm}$ would suggest that Class I and possibly Class II tryptophans dominate the fluorescence spectrum of SoPIP2;1. This automatic analysis of PDB-files is most likely not applicable for tryptophans that are expected to interact with detergents in a micelle, since the micelle is not included in the structure file. However, it should be relevant for buried residues, like tryptophan 79 situated at the center of the tetramer (Figure 8). Based on the closed tetrameric structure (4JC6, omitting detergents or metal atoms) this residue belongs to Class $\mathrm{S}$ with an expected emission maximum range between 321 and $325 \mathrm{~nm}$, and is therefore not likely to contribute much to the intensity at $335 \mathrm{~nm}$.

As speculated above the mercury activation may be achieved by stabilizing an open conformation and interestingly the positions of tryptophans 35 and 38 differ in the open and closed structure (Törnroth-Horsefield et al., 2006). In the closed structure tryptophan 35 is potentially in a hydrophobicpolar interface and close to the C-terminus of the next monomer. In the open structure of SoPIP2;1 tryptophan 35 is repositioned and in addition this conformation is expected to have released the C-terminus, which could have an effect on the environment of tryptophan 35 and therefore quench its fluorescence.

\section{Mercury Binding Site}

In a mutational study of SoPIP2;1 by Frick et al. (2013) it was found that mercury activation is independent of the presence 


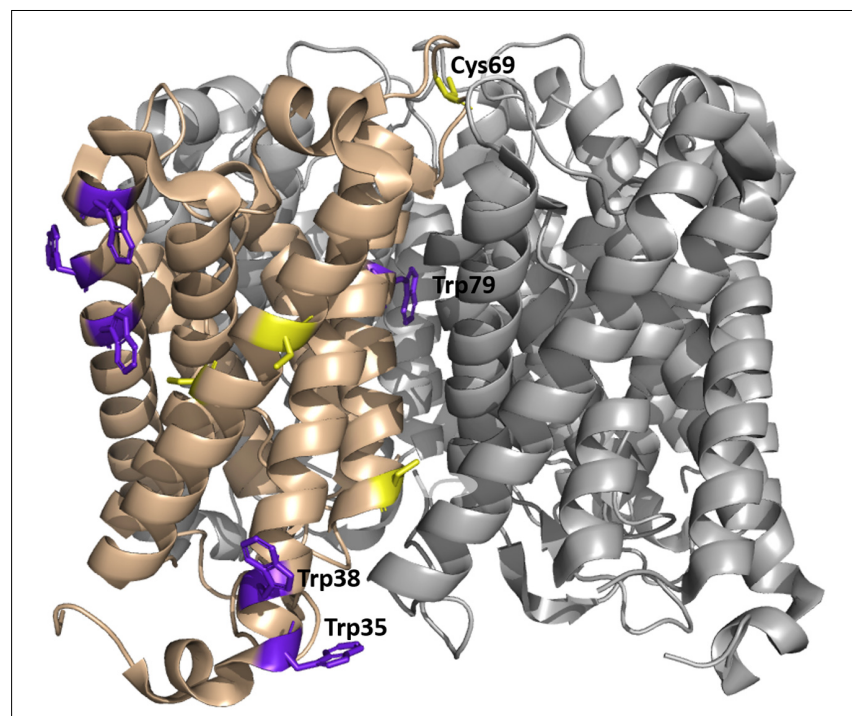

FIGURE 8 | Tetrameric structure of WT SoPIP2;1 in a closed conformation (PDB ID 1Z98) showing positions of tryptophan and cysteine residues. Each monomer exposes six tryptophans on its outside (purple); four of them (left in the picture) are expected to be in a non-polar environment (detergent micelle in buffer or a lipid membrane), one (tryptophan 79 ) is positioned in the tetrameric center and only tryptophan 35 is exposed to mobile waters, sitting at the beginning of helix 1 and close to the end of helix 6 and the $\mathrm{C}$-terminus of the next monomer. Four cysteines are present in each monomer, two (left in the picture) are situated close to the tetramer equator, one is positioned in the monomer interface close to the cytosolic side, and cysteine 69 is highly accessible at the periplasmic side of the tetrameric center.

of cysteines. They were able to determine a structure at $2.15 \AA$ with bound mercury and cadmium, in a closed conformation. Additional to the expected binding to cysteines and the known $\mathrm{Ca}^{2+}$ site, a new metal binding site was reported. It is described as a second binding site for cadmium, but given the comparable size of the cations and similar preference for hexagonal coordination, one could imagine that also mercury has a potential to bind.
The metal seen at this site interacts with a carbonyl (A267) from the C-terminal region (Figure 9A). The carbonyl of A267 is actually occupying the same space as the carbonyl of A266 in the first closed structure of SoPIP2;1 (Törnroth-Horsefield et al., 2006), and consequently there is a different position and orientation of preceding residues up to the end of helix 6 . This distinctive change in the structure does not change the averaged static view on the C-terminal regions, which overall are located at the interfaces of the monomers in a closed conformation. Still, the binding strength might be reduced and thereby lower the probability to find this conformation in solution. The conformation of the protein when incubated in $\beta$-mercaptoethanol after binding mercury does not change within seconds (Figure 5B). We expect that mercury binding or chelating is much faster. Thus, the observed time dependence could be a result of the restructuring of the C-terminal end. If the activation is caused by destabilization of the C-terminal stretch, the effect could be reduced in situations where it is already destabilized for other reasons. This is observed, when comparing the permeability of an untagged SoPIP2;1 WT and the permeability of the same isoform that ends with a c-myc epitope followed by a hexa-his-tag. The basal (i.e., not fully activated) permeability is higher for the tagged protein, seemingly because the closed conformation of the unphosphorylated C-terminal end is less stabilized (data not shown). It should not go unnoted that similar conclusions were drawn before (Nyblom, 2008, Ph.D. thesis, paper III, page 5). When incubated with mercury the specific water permeability, of both the tagged and untagged protein, levels out at approximately the same permeability values. Conclusively, destabilization by binding of mercury overlaps the destabilization caused by the tag, suggesting that the gate is close to its maximum opening probability in the mercury activated state. Frick et al. (2013) suggested an indirect activation of SoPIP2; 1 via a mercury induced change in membrane properties. This possibility cannot be ruled out, but the relatively low $\mathrm{EC}_{50}$ of about $4 \mu \mathrm{M}$, which is about 10 -fold lower than the $\mathrm{IC}_{50}$ for HsAQP5 in proteoliposomes with the same lipid composition (Horsefield et al., 2008), and the correlation with the quenching
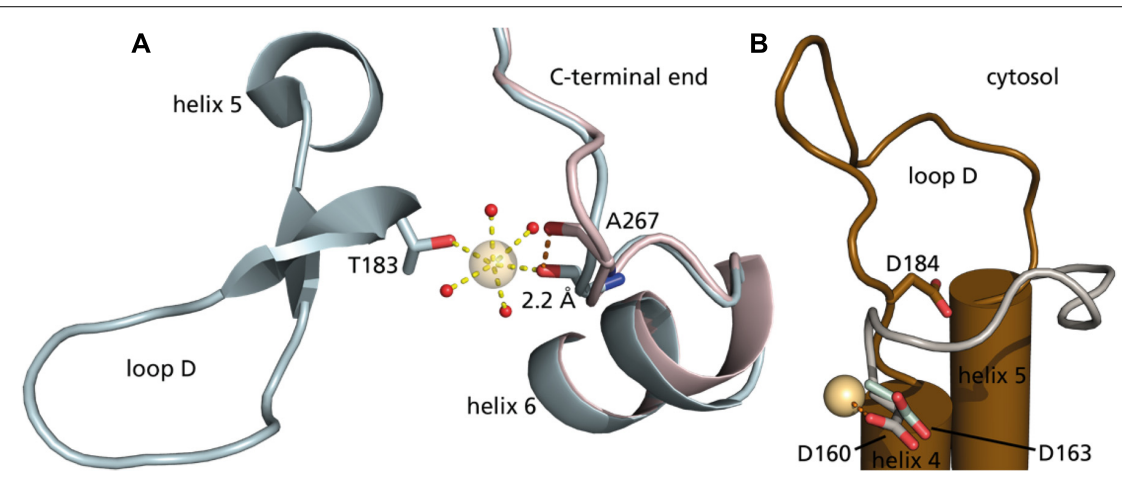

FIGURE 9 | The second metal binding site in SoPIP2;1. (A) Cytoplasmic view on two closed structures of SoPIP2;1, with mercury (PDB ID 4JC6) in blue and without mercury (PDB ID 1Z98) in pink. Alanine 267 is shifted $2.2 \AA$ to coordinate the additional heavy metal site. The heavy atom is further coordinated in this structure by threonine 183 at the beginning of loop $D$ and four water molecules (oxygens in red). (B) Comparison of conserved aspartate in the open structure of SoPIP2;1 (D184, brown, one monomer of PDB ID 2B5F) with aspartates of AtTIP2;1 (D163, light green, 5I32) and BtAQP1 (D160, gray, 1J4N). The carboxyl group of D160 from the aligned bovine structure is close $(2.7 \AA)$ to the heavy metal binding site in 4JC6. 
of fluorescence in micelles, would argue for a direct binding to and activation of SoPIP2;1.

Here, we describe the activation by mercury as a result of moving serine 274 away to leave space for leucine 197 in an open position, to promote the conformation. However, could mercury further stabilize the open conformation? As mercury is positively charged, we looked for a negatively charged residue in the vicinity. There is an aspartate in loop $\mathrm{D}$, which is conserved among water-specific AQPs from both plants and animals. This residue approaches the binding site in the open conformation (Figure 9B), but the distance is still too far. Comparison to other water-specific AQPs suggests that the aspartate could come close enough to bind mercury and thereby stabilize the D-loop in an open position. Although the aspartate corresponding to aspartate 184 in SoPIP2;1 is well conserved not only among PIPs, there is considerable sequence variation at the position aligned to alanine 267 of SoPIP2;1 even within the PIP2 group, making it difficult discern if the proposed activation mechanism is valid for other PIPs. However, the fact that it is the back bone carbonyl of this residue that contributes to the metal binding site, and the general observation that structure is more conserved than sequence, argues that the binding site and the suggested mechanism may be relevant also for other PIP2s of terrestrial plants. It should be noted that the plant plasma membrane has a different lipid composition and is much more complex than the liposomes of $E$. coli lipids used here. Hence, the relevance of the demonstrated mercury activation remains to be shown in a physiological context where indirect effects by mercury via interacting lipids or proteins may override a direct activation.

\section{Is There a Central Pore?}

The functionality of the protein is determined by stabilization of certain conformational states and thereby dependent on the availability of certain interacting residues, not only within a monomer, but also with neighboring monomers. This has already been described for many proteins and underlines that proteins evolve based on their final oligomeric structure. This brings us back to cysteine 69 , which is found to be strongly conserved among PIPs and covalently links the monomers but does not add much thermodynamic stability. Interaction studies with PIPs in maize demonstrated that expression rate and localization is also independent of disulfide bond formation (Bienert et al., 2012). Taking only our own results into account, one could suggest the prevention of water leakage through the tetrameric center, but this is inconclusive with the mutational study of maize PIPs measured in oocytes. As the utilized maize PIP2 is very similar to SoPIP2;1, the most prominent difference between these studies is the protein environment. As several AQP structures, including SoPIP2;1, have presented a detergent or a lipid molecule in the tetrameric center (Jiang et al., 2006; Horsefield et al., 2008; Frick et al., 2013), one could assume the presence of a lipid molecule at these sites in the native environment to avoid water or even proton leakage. In this study, the protein was heterologously expressed and extracted from the hydrophobic environment by a detergent. These detergent molecules are capable of removing most or all of the lipid molecules bound to the protein. Additionally, the protein-lipid mixture was thoroughly dialyzed to reconstitute this protein in liposomes, potentially removing all detergent molecules. Thus cysteine 69 mutants could leak water through a central pore. As other AQP isoforms without this cysteine have also evolved to be proton tight, there is probably a different reason for the high degree of conservation. We therefore agree with Bienert et al. (2012) that disulfide bonds between monomers result in a higher kinetic stability and thereby stabilize the oligomeric state of the protein, which may comprise certain functions in planta.

\section{CONCLUSION}

In agreement with studies on maize PIPs we show that disulfide bridges stabilize the oligomeric state of SoPIP2;1, but contrary to results on maize PIPs we find that mutations preventing the disulfide bridge increase the permeability. Furthermore, the permeability of both WT and mutant protein can be enhanced by mercury and based on quenching of the intrinsic tryptophan fluorescence by mercury we speculate on a binding site, possibly responsible for both effects of this heavy metal. Mercury has been regarded as general inhibitor for AQPs and therefore used to classify observed in vivo phenomena as being "AQP dependent." The sole possibility that some AQPs under certain circumstances can be activated by mercury puts these conclusions into a new perspective. Mercury is not a physiological relevant ligand to regulate protein function, but presents a tool to study functional properties of the gating mechanism of PIPs and thereby potentially revealing new opportunities to modulate water homeostasis in plants.

\section{MATERIALS AND METHODS}

\section{Overexpression and Purification of Cysteine Mutants of SoPIP2;1}

Cysteine mutants of SoPIP2;1 were generated by using Quickchange site directed mutagenesis kit (Stratagene), using the plasmid pPICZB-SoPIP2;1 (WT) with C-terminal His-tag as a template (Karlsson et al., 2003). Mutations were confirmed by DNA sequencing (Eurofins MWG operon). Mutants were overexpressed in methylotropic yeast Pichia pastoris as previously described (Karlsson et al., 2003) for WT SoPIP2;1 purification. Similar procedure was followed for membrane preparation and purification of mutated proteins as described previously (Karlsson et al., 2003) with a minor change; the detergent used in the present study is $n$-Octyl- $\beta$-D-Glucopyranoside (OG; Affymetrix, O311).

\section{Electrophoresis}

The purified SoPIP2;1 (WT) was incubated at room temperature for varying time periods, with different concentration of dithiothreitol (DTT) or $\beta$-mercaptoethanol to reduce the dimeric form into monomers. After incubation with reducing agent the sample loading buffer (125 mM Tris- $\mathrm{HCl}, \mathrm{pH} 6.8,20 \%$ glycerol, $4 \%$ SDS) was mixed with protein sample and further 
incubated for $10 \mathrm{~min}$ at room temperature. To monitored the effect of reducing agent concentration and incubation time, the oligomeric forms of the protein were resolved by SDS-PAGE (12\%) and visualized by staining with coomassie brilliant blue R250 (Laemmli, 1970).

In order to compare the SDS-PAGE profile of the WT and mutant proteins (Figure 3), the protein was directly mixed with sample loading buffer (as mentioned above) supplemented with $10 \% \beta$-mercaptoethanol and incubated for $10 \mathrm{~min}$ at room temperature, before resolving on SDS-PAGE (12\%).

\section{Circular Dichroism (CD) Spectroscopy}

Far-UV CD spectra were measured for the WT SoPIP2;1 and the mutants using a Jasco J-720 spectrometer (Jasco, Tokyo, Japan). Spectra were recorded at $25-95^{\circ} \mathrm{C}$ (with $5^{\circ} \mathrm{C}$ interval) between $250 \mathrm{~nm}$ and $190 \mathrm{~nm}$ at $20 \mathrm{~nm} / \mathrm{min}$ as an average of three scans with a response time $8 \mathrm{~s}$ and a data pitch of $0.1 \mathrm{~nm}$. Baselines were collected in the same manner on the buffer solution, and spectra were baseline corrected (Galka et al., 2008; Plasencia et al., 2011).

Mean residue ellipticity (MRE, $[\theta]_{\mathrm{M}} \times 10^{-3} \mathrm{deg} \mathrm{cm}^{2} \mathrm{dmol}^{-1}$ ) was calculated by using Eq. (1).

$$
[\theta]_{M}=M \times \theta /(10 \times 1 \times c \times n)
$$

where $\mathrm{M}$ is the molecular weight of protein (e.g., $32512 \mathrm{~g} / \mathrm{mol}$ ), $\theta$ is the measured ellipticity in millidegrees, 1 is the cell path length, $\mathrm{c}$ is the concentration in $[\mathrm{g} / \mathrm{l}]$, and $\mathrm{n}$ is the number of residues (303).

The MRE at $222 \mathrm{~nm}$ was plotted over temperature. For curve fitting, following Boltzmann sigmoidal equation was used:

$$
Y_{\text {obs }}=Y_{\text {native }}-\frac{Y_{\text {denatured }}-Y_{\text {native }}}{1+\mathrm{e}^{T_{1 / 2}-\frac{T}{m}}}
$$

Where $\mathrm{Y}_{\mathrm{obs}}$ is the MRE, $\mathrm{T}_{1 / 2}$ is the temperature at which MRE is halfway between native and denatured state, $\mathrm{m}$ is the slope of the curve. Data was analyzed using Prism (Graphpad software, Inc.).

\section{Reconstitution into Liposomes}

E. coli POLAR lipids (Avanti) provided in chloroform were dried with $\mathrm{N}_{2}$ for $4 \mathrm{~h}$ and kept at $-20^{\circ} \mathrm{C}$ until use. For reconstitution the lipids were solubilized with $10 \%$ OG in dialyze buffer $(20 \mathrm{mM}$ Tris $\mathrm{pH} 8,100 \mathrm{mM} \mathrm{NaCl}, 0.003 \% \mathrm{NaN}_{2}, 2 \mathrm{mM}$ DTT) for concentration of $4 \mathrm{mg} / \mathrm{mL}$ and aliquoted. Proteins were added and solution was mixed thoroughly. Lipid-protein mix was diluted with dialyze buffer to $2 \mathrm{mg} / \mathrm{mL}$ lipids and $66 \mu \mathrm{g} / \mathrm{mL}$ proteins (LPR30) and dialyzed using a membrane with $6-8 \mathrm{kDa}$ cut-off (Spectrum Laboratories) against dialyze buffer for 7 days at room temperature.

\section{Water Conductivity}

Liposomes were extruded 11 times with a pore size of $200 \mathrm{~nm}$ (Avestin) and their resulting average radius was determined by dynamic light scattering DLS (Malvern Zetasizer). Samples were diluted with dialyze buffer (with or without mercury) to
$0.2 \mathrm{mg} / \mathrm{mL}$ lipids. To show that activation of the protein is reversible, a sample incubated for $30 \mathrm{~min}$ with $1 \mathrm{mM} \mathrm{HgCl}_{2}$, $2 \mathrm{mM} \beta$-mercaptoethanol was added and incubated at least $30 \mathrm{~min}$ further prior to the activity assay. Water transport activity was measured by stopped-flow with a hyper-osmolar gradient of $100 \mathrm{mM}$ sorbitol using Hi-Tech stopped-flow device at a volume of $150 \mu \mathrm{L}$ per shot. Rise in scattering upon shape change due to water transport out of the vesicles was observed at $90^{\circ}$ angle at a wavelength of $500 \mathrm{~nm}$. Unless mentioned otherwise, single exponential functions were fitted to 10 to 17 individual curves by the software Kinetic Studio (TgK Scientific Limited 2010). Total water permeability $P_{\mathrm{f}}$ is used to calculate individual $p_{\mathrm{f}}$-values by multiplying relative $P_{\mathrm{f}}\left[P_{\mathrm{f}}\right.$ (proteoliposome) $-P_{\mathrm{f}}$ (control liposomes)] with the surface area of the liposome and dividing with the number of monomers per vesicle.

$$
\begin{gathered}
P_{\mathrm{f}}=\mathrm{k} \cdot \frac{\mathrm{V}_{0}}{\mathrm{~A} \cdot \mathrm{V}_{\mathrm{w}} \cdot \mathrm{c}_{\mathrm{out}}} \\
p_{\mathrm{f}}=\frac{\left(P_{\mathrm{f}}-P_{\mathrm{f}, \text { control }}\right) \cdot \mathrm{A}}{\# \text { monomers }}
\end{gathered}
$$

Protein concentration was analyzed by Western-blot using tetraHis antibody, and vesicle concentration was calculated assuming no lipid loss during dialysis and an area of $0.52 \mathrm{~nm}^{2}$ per lipid molecule (for a monolayer). To construct a dose response curve of mercury activation, single exponential rates from 10 to 16 stopped-flow measurements were averaged per samples exposed to different mercury concentration; each average is presented with their standard deviations. Curve fitting was done employing a Hill-function.

\section{Statistical Analyses}

The significance was analyzed in Prism (Graphpad software, Inc.), using the unpaired $t$-test with Welch's correction and twotailed $P$-values.

\section{Tryptophan Fluorescence}

Fluorescence was measured with His-tag purified and desalted protein in Buffer A supported by $0.8 \%$ OG. Monochromatic light at a wavelength of $280 \mathrm{~nm}$ was used for excitation while scans were done from 300 to $380 \mathrm{~nm}$ at a speed of $100 \mathrm{~nm} / \mathrm{min}$ and a data pitch of $0.5 \mathrm{~nm}$. Fifteen scans were accumulated to reduce noise. For kinetic experiments, mercury chloride to a final concentration of $200 \mu \mathrm{M}$ was added and briefly mixed before starting scans. First, single records were done with a delay of $1 \mathrm{~s}$, resulting in one plot every $49 \mathrm{~s}$. The averages of five data points were used to determine height of the curve maximum. After incubating for $25 \mathrm{~min}$, another accumulated spectrum was recorded. Sequentially, $\beta$-mercaptoethanol to a final concentration of $1 \mathrm{mM}$ was added to reverse the binding, followed by the same measurement procedure as before.

For dose-response experiments, the sample was pre-incubated with mercury chloride at room temperature for $15 \mathrm{~min}$ before 
recording the fluorescence between 310 and $400 \mathrm{~nm}$. Three scans were accumulated to reduce the noise and the measurement was repeated tree times. The emission data at $335 \mathrm{~nm}$ were fitted with four parameters to sigmoidal dose response equation using Prism version 6.00 (GraphPad Software, La Jolla, CA, USA) to estimate the $\mathrm{EC}_{50}$ value.

\section{AUTHOR CONTRIBUTIONS}

AK and SS contributed equally to this work, did all the technical work, and analyzed the data. PK and UJ conceived the project. AK drafted the manuscript and all authors contributed to writing of the final article.

\section{REFERENCES}

Abascal, F., Irisarri, I., and Zardoya, R. (2014). Diversity and evolution of membrane intrinsic proteins. Biochim. Biophys. Acta 1840, 1468-1481. doi: 10.1016/j.bbagen.2013.12.001

Alexandersson, E., Danielson, J. A., Råde, J., Moparthi, V. K., Fontes, M., Kjellbom, P., et al. (2010). Transcriptional regulation of aquaporins in accessions of Arabidopsis in response to drought stress. Plant J. 61, 650-660. doi: 10.1111/j.1365-313X.2009.04087.x

Alexandersson, E., Fraysse, L., Sjövall-Larsen, S., Gustavsson, S., Fellert, M., Karlsson, M., et al. (2005). Whole gene family expression and drought stress regulation of aquaporins. Plant Mol. Biol. 59, 469-484. doi: 10.1007/s11103005-0352-1

Bienert, G. P., Cavez, D., Besserer, A., Berny, M. C., Gilis, D., Rooman, M., et al. (2012). A conserved cysteine residue is involved in disulfide bond formation between plant plasma membrane aquaporin monomers. Biochem. J. 445, 101111. doi: 10.1042/bj20111704

Clarke, J., and Fersht, A. R. (1993). Engineered disulfide bonds as probes of the folding pathway of barnase: increasing the stability of proteins against the rate of denaturation. Biochemistry 32, 4322-4329. doi: 10.1021/bi0006 $7 \mathrm{a} 022$

Danielson, J. A. H., and Johanson, U. (2010). "Phylogeny of Major Intrinsic Proteins," in Mips and Their Role in the Exchange of Metalloids, eds T. P. Jahn and G. P. Bienert (New York, NY: Springer), 19-32.

Frick, A., Järvå, M., Ekvall, M., Uzdavinys, P., Nyblom, M., and TörnrothHorsefield, S. (2013). Mercury increases water permeability of a plant aquaporin through a non-cysteine-related mechanism. Biochem. J. 454, 491-499. doi: 10.1042/BJ20130377

Fu, D., Libson, A., Miercke, L. J., Weitzman, C., Nollert, P., Krucinski, J., et al. (2000). Structure of a glycerol-conducting channel and the basis for its selectivity. Science 290, 481-486. doi: 10.1126/science.290. 5491.481

Galka, J. J., Baturin, S. J., Manley, D. M., Kehler, A. J., and O’neil, J. D. (2008). Stability of the glycerol facilitator in detergent solutions. Biochemistry 47, 3513-3524. doi: 10.1021/bi7021409

Horsefield, R., Nordén, K., Fellert, M., Backmark, A., Törnroth-Horsefield, S., Terwisscha van Scheltinga, A. C., et al. (2008). High-resolution x-ray structure of human aquaporin 5. Proc. Natl. Acad. Sci. U.S.A. 105, 13327-13332. doi: 10.1073/pnas.0801466105

Hukin, D., Doering-Saad, C., Thomas, C. R., and Pritchard, J. (2002). Sensitivity of cell hydraulic conductivity to mercury is coincident with symplasmic isolation and expression of plasmalemma aquaporin genes in growing maize roots. Planta 215, 1047-1056. doi: 10.1007/s00425-0020841-2

Jiang, J., Daniels, B. V., and Fu, D. (2006). Crystal structure of AqpZ tetramer reveals two distinct Arg-189 conformations associated with water permeation through the narrowest constriction of the water-conducting channel. J. Biol. Chem. 281, 454-460. doi: 10.1074/jbc.M508926200

\section{FUNDING}

This work was supported by the Swedish Research Council.

\section{ACKNOWLEDGMENT}

We thank Adine Karlsson for technical assistance and Dr S. Raza Haq for help with statistical analyses.

\section{SUPPLEMENTARY MATERIAL}

The Supplementary Material for this article can be found online at: http://journal.frontiersin.org/article/10.3389/fpls.2016.01249

Johanson, U., Karlsson, M., Johansson, I., Gustavsson, S., Sjövall, S., Fraysse, L., et al. (2001). The complete set of genes encoding major intrinsic proteins in Arabidopsis provides a framework for a new nomenclature for major intrinsic proteins in plants. Plant Physiol. 126, 1358-1369. doi: 10.1104/pp.126. 4.1358

Johansson, I., Karlsson, M., Shukla, V. K., Chrispeels, M. J., Larsson, C., and Kjellbom, P. (1998). Water transport activity of the plasma membrane aquaporin PM28A is regulated by phosphorylation. Plant Cell 10, 451-459. doi: 10.1105/tpc.10.3.451

Johansson, I., Larsson, C., Ek, B., and Kjellbom, P. (1996). The major integral proteins of spinach leaf plasma membranes are putative aquaporins and are phosphorylated in response to $\mathrm{Ca}^{2+}$ and apoplastic water potential. Plant Cell 8, 1181-1191. doi: 10.1105/tpc.8.7.1181

Karlsson, M., Fotiadis, D., Sjövall, S., Johansson, I., Hedfalk, K., Engel, A., et al. (2003). Reconstitution of water channel function of an aquaporin overexpressed and purified from Pichia pastoris. FEBS Lett. 537, 68-72. doi: 10.1016/S00145793(03)00082-6

Kirscht, A., Kaptan, S. S., Bienert, G. P., Chaumont, F., Nissen, P., de Groot, B. L., et al. (2016). Crystal structure of an ammonia-permeable aquaporin. PLoS Biol. 14:e1002411. doi: 10.1371/journal.pbio.100 2411

Kukulski, W., Schenk, A. D., Johanson, U., Braun, T., de Groot, B. L., Fotiadis, D., et al. (2005). The 5A structure of heterologously expressed plant aquaporin SoPIP2;1. J. Mol. Biol. 350, 611-616. doi: 10.1016/j.jmb.2005. 05.001

Laemmli, U. K. (1970). Cleavage of structural proteins during the assembly of the head of bacteriophage T4. Nature 227, 680-685. doi: 10.1038/227 $680 \mathrm{a} 0$

Lakowicz, J. R. (ed.) (2006). Principles of Fluorescence Spectroscopy. Berlin: Springer.

Nyblom, M. (2008). Water Transport Regulation: Biochemical and Structural Analyses of Eukaryotic Aquaporins. Ph.D. thesis, Chalmers University of Technology, Gothenburg.

Nyblom, M., Frick, A., Wang, Y., Ekvall, M., Hallgren, K., Hedfalk, K., et al. (2009). Structural and functional analysis of SoPIP2;1 mutants adds insight into plant aquaporin gating. J. Mol. Biol. 387, 653-668. doi: 10.1016/j.jmb.2009. 01.065

Plasencia, I., Survery, S., Ibragimova, S., Hansen, J. S., Kjellbom, P., HelixNielsen, C., et al. (2011). Structure and stability of the spinach aquaporin SoPIP2;1 in detergent micelles and lipid membranes. PLoS ONE 6:e14674. doi: 10.1371/journal.pone.0014674

Preston, G. M., Jung, J. S., Guggino, W. B., and Agre, P. (1993). The mercurysensitive residue at cysteine 189 in the CHIP28 water channel. J. Biol. Chem. 268, 17-20.

Shen, C., Menon, R., Das, D., Bansal, N., Nahar, N., Guduru, N., et al. (2008). The protein fluorescence and structural toolkit: database and programs for the analysis of protein fluorescence and structural data. Proteins 71, 1744-1754. doi: $10.1002 /$ prot. 21857 
Sui, H., Han, B. G., Lee, J. K., Walian, P., and Jap, B. K. (2001). Structural basis of water-specific transport through the AQP1 water channel. Nature 414, 872-878. doi: $10.1038 / 414872 \mathrm{a}$

Törnroth-Horsefield, S., Wang, Y., Hedfalk, K., Johanson, U., Karlsson, M., Tajkhorshid, E., et al. (2006). Structural mechanism of plant aquaporin gating. Nature 439, 688-694. doi: 10.1038/nature04316

Yang, B., and Verkman, A. S. (1997). Water and glycerol permeabilities of aquaporins 1-5 and MIP determined quantitatively by expression of epitopetagged constructs in Xenopus oocytes. J. Biol. Chem. 272, 16140-16146. doi: $10.1074 /$ jbc. 272.26 .16140
Conflict of Interest Statement: The authors declare that the research was conducted in the absence of any commercial or financial relationships that could be construed as a potential conflict of interest.

Copyright (c) 2016 Kirscht, Survery, Kjellbom and Johanson. This is an open-access article distributed under the terms of the Creative Commons Attribution License (CC BY). The use, distribution or reproduction in other forums is permitted, provided the original author(s) or licensor are credited and that the original publication in this journal is cited, in accordance with accepted academic practice. No use, distribution or reproduction is permitted which does not comply with these terms. 